\title{
In-situ Open Cell TEM/STEM Environmental Study of Iron Oxides Nanoparticles and Sample-Beam Interaction in $\mathrm{O}_{2}$ gas
}

\author{
Leonardo Lari $^{1,2}$, Stephan Steinhauer ${ }^{3}$ and Mukhles Sowwan ${ }^{3}$ and Vlado K Lazarov ${ }^{1,2}$ \\ 1. University of York, Department of Physics, Heslington, York, YO10 5DD, North Yorkshire, UK \\ 2. University of York, The York-JEOL Nanocentre, Heslington, Science Park, York, YO10 5BR, UK \\ 3. Nanoparticles by Design Unit, OIST Graduate University, Okinawa 904-0495, Japan
}

The study of Iron Oxides Nanoparticles (NPs) has burgeoned during the last decades due to the large number of applications of such structures in catalysis and nanotechnology, going from water purification and ferrofluids, to hyperthermia treatments of tumors as well as MRI contrast enhancement [1-3].

In-situ environmental (scanning) transmission electron microscopy E-(S)TEM [4] gives a new opportunity of studying oxidation and reduction of nanoparticles under controlled atmosphere and temperature at the nanoscale. This technique provides the possibility of imaging NPs and to follow their structural transformations as a function of temperature and gas pressure. In addition to structural information, electron diffraction, energy dispersive x-ray analysis as well as electron energy loss spectroscopy can provide chemical information at the same time.

Samples for in-situ investigation were prepared by using a glow discharge sputter coater with a $\mathrm{Fe}$ target. The sputtering was done directly either on $\mathrm{C}$ films supported by TEM copper grids, SiN membranes as well as MEMS heating chips. Two environmental microscopes were used: an FEI/Thermo environmental Titan TEM operating at $300 \mathrm{KeV}$ at OIST (Japan) equipped with a postcolumn Gatan electron energy loss spectrometer, and the York (UK) in-house modified in-situ E(S)TEM JEOL 2200FS 200kV microscope[5] equipped with a 100mm2 EDX spectrometer (Thermo) and an in-column "Omega type" electron energy filter. Conventional heating holders (Gatan model 628) were used for the analyses as well as MEMS chip based holders (Dens solution, Wildfire).

The pre-sputtered thin films were annealed in-situ up to 700C in vacuum to study the formation of NPs within the microscope column. TEM HREM imaging showed the nanoparticle crystals were compatible with both $\mathrm{Fe}_{3} \mathrm{O}_{4}$ (Magnetite) and $\gamma-\mathrm{Fe}_{2} \mathrm{O}_{3}$ (Maghemite) phases (Fig.1). Due to the close lattice spacing of these two phases, imaging cannot distinguish between the two structures. For this reason we used zeroloss energy filtered electron diffraction analysis and we were able to revel that the NPs phase was Magnetite.

The effects of the STEM probe/sample interaction were also studied. We found that oxidation is enhanced by the presence of the beam during gas exposure to the sample. In particular, oxidation in presence of the STEM probe facilitates the formation of Kirkendall voids not observed when gas exposure is done with the beam off (Fig. 2).

The Fe-O phase diagram was then explored by varying temperature and/or oxygen partial pressure. We found that at $800 \mathrm{C}$ temperature Magnetite and $\alpha-\mathrm{Fe}_{2} \mathrm{O}_{3}$ (Hematite) NP phases were coexisting in vacuum. By decreasing the temperature (300C) and increasing the Oxygen partial pressure in the range $10^{-2} \mathrm{~Pa}$ to $1 \mathrm{~Pa}$ Magnetite nanoparticles where found to further oxidize at the edges while Hematite nanoparticles remained stable (Fig. 2, right). 


\section{References:}

[1] B D Terris and T Thomson, J. Phys. D 38 (2005) R199.

[2] H. Galvis et al. Science 335 (2012) 835.

[3] Q A Pankhurst et al., J. Phys. D 42 (2009) 224001.

[4] P L Gai, Topics in Catalysis 21 (2002) 161

[5] E. D. Boyes, M. R. Ward, L. Lari, and P. L. Gai, Annalen der Physik 525 (2013) 423

[6] The authors acknowledge Profs. Ed Boyes and P Gai for the E(S)TEM in-situ development at York.

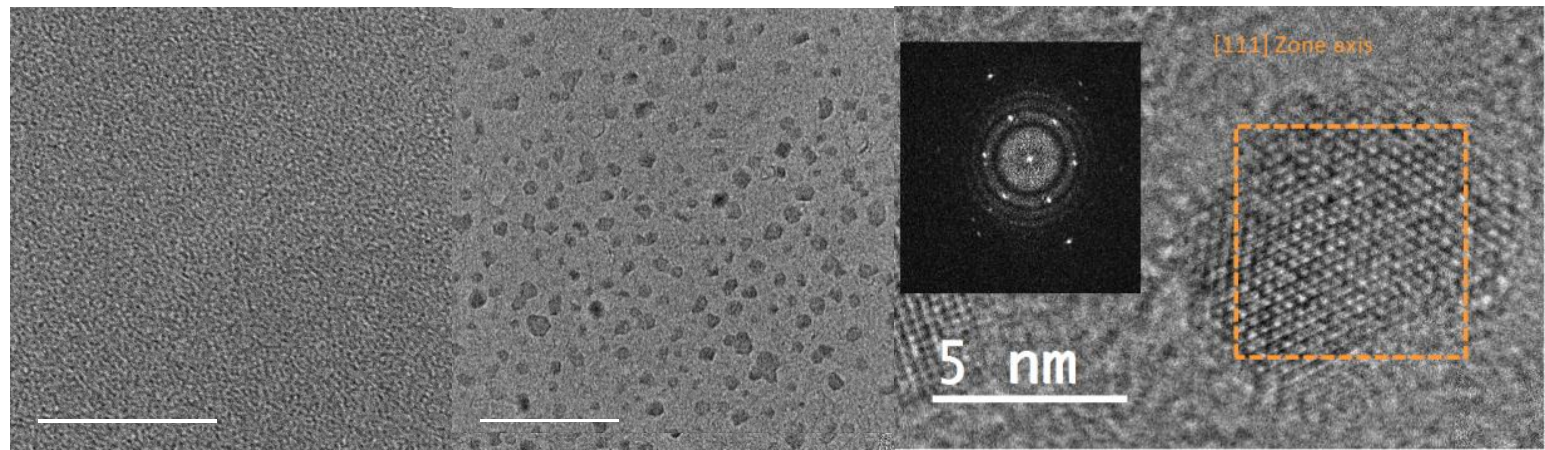

Figure 1. (left) BF image as sputtered film, (center) in-situ nanoparticle formation at 700C (right) HREM image with inset FFT of a structure compatible with $\mathrm{Fe}_{3} \mathrm{O}_{4}$ and $\gamma-\mathrm{Fe}_{2} \mathrm{O}_{3}$.

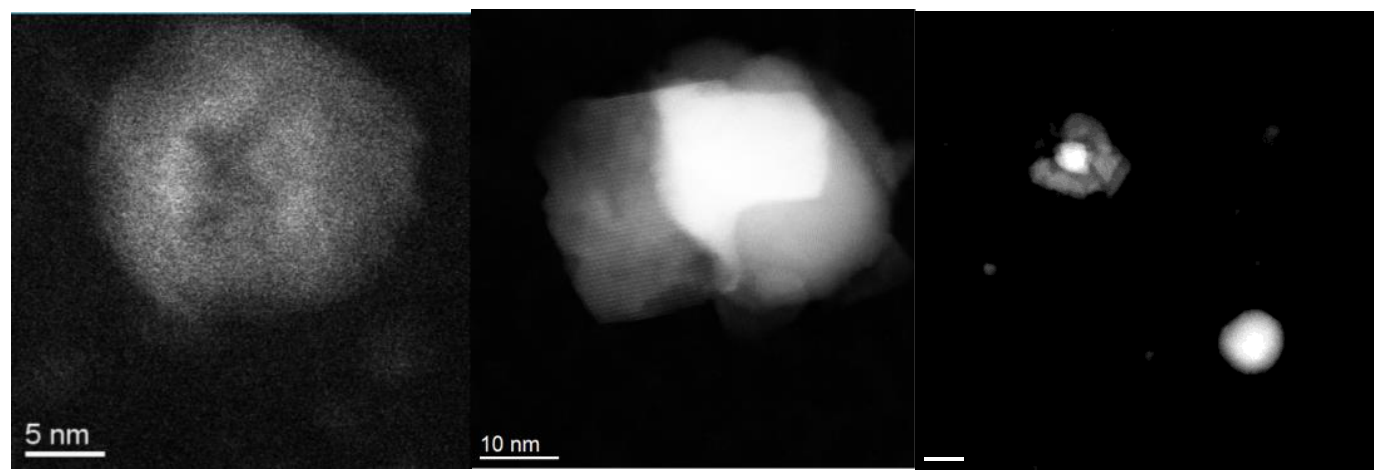

Figure 2. HAADF STEM images of: (left) a NP oxidized while scanning the STEM probe showing a Kirkendal effect; (center) a NP oxidized with the beam off; (right) Oxidation of $\mathrm{Fe}_{3} \mathrm{O}_{4} \mathrm{NPs}$ and stability of Hematite $\left(\alpha-\mathrm{Fe}_{2} \mathrm{O}_{3}\right)$ NPs at 300C. 